\title{
Recent results on dual readout in calorimetry from the DREAM Collaboration
}

\section{Francesco LACAVA*}

Dip. Fisica Università Sapienza / INFN Roma

E-mail: francesco.lacavaeromal.infn.it

on behalf of the DREAM COLLABORATION.

The energy resolution of the hadronic calorimeters is mostly limited by the fluctuation of the electromagnetic component in the development of the shower. The dual readout technique proposed by DREAM aims to correct event by event for this fluctuation measuring at the same time scintillation and Cherenkov light. In the last years the DREAM Collaboration has performed several tests to exploit the dual readout technique in calorimeters. Many types of crystals ( $\mathrm{PbWO}, \mathrm{BGO}$, BSO, etc.) have been exposed in test beams to evaluate the Cherenkov yield and how to optimize the light collection. The extention of the dual readout to crystals is in fact very important when an electromagnetic calorimeter composed by crystals is followed by an hadronic calorimeter. The possibility to use tiles of absorber interleaved with tiles of quartz and scintillator for the dual readout has also been considered. At present the DREAM Collaboration is testing new calorimeters with scintillating and clear fibers.

XXIst International Europhysics Conference on High Energy Physics

21-27 July 2011

Grenoble, Rhône-Alpes France

\footnotetext{
* Speaker.
} 


\section{Introduction.}

The energy resolution in the hadronic calorimeters is determined by fluctuations in the development of the shower. In non compensating hadronic calorimeters the dominant contribution is the fluctuation of the electromagnetic shower fraction. The dual readout method proposed by DREAM aims to correct event by event for this fluctuation measuring at the same time the signals from scintillation (S) due to ionization from all charged particles and from the Cherenkov light (C) radiated only from relativistic particles (mainly secondaries in the electromagnetic component).

The electromagnetic fraction $f_{e m}$ can be determined from the ratio of the two signals:

$$
\frac{C}{S}=\frac{f_{e m}+\lambda_{C}\left(1-f_{e m}\right)}{f_{e m}+\lambda_{S}\left(1-f_{e m}\right)}
$$

where $\lambda_{C}$ and $\lambda_{S}$ are the ratios of the responses to an intrinsic (that is without any e.m. component) hadronic shower and to an electromagnetic shower in the measurements of Cherenkov and scintillation signals.

This technique was proven in the DREAM calorimeter [1] where the two active media are: scintillating fibers to measure the $\mathrm{dE} / \mathrm{dx}$ ionization of all the charged particles, and clear (quartz or plastic) fibers to measure the Cherenkov light. In fig. 1 are reported the results for a $200 \mathrm{GeV}$ "jet" (pion interacting in a target in front of the calorimeter) before and after the $C / S$ correction.
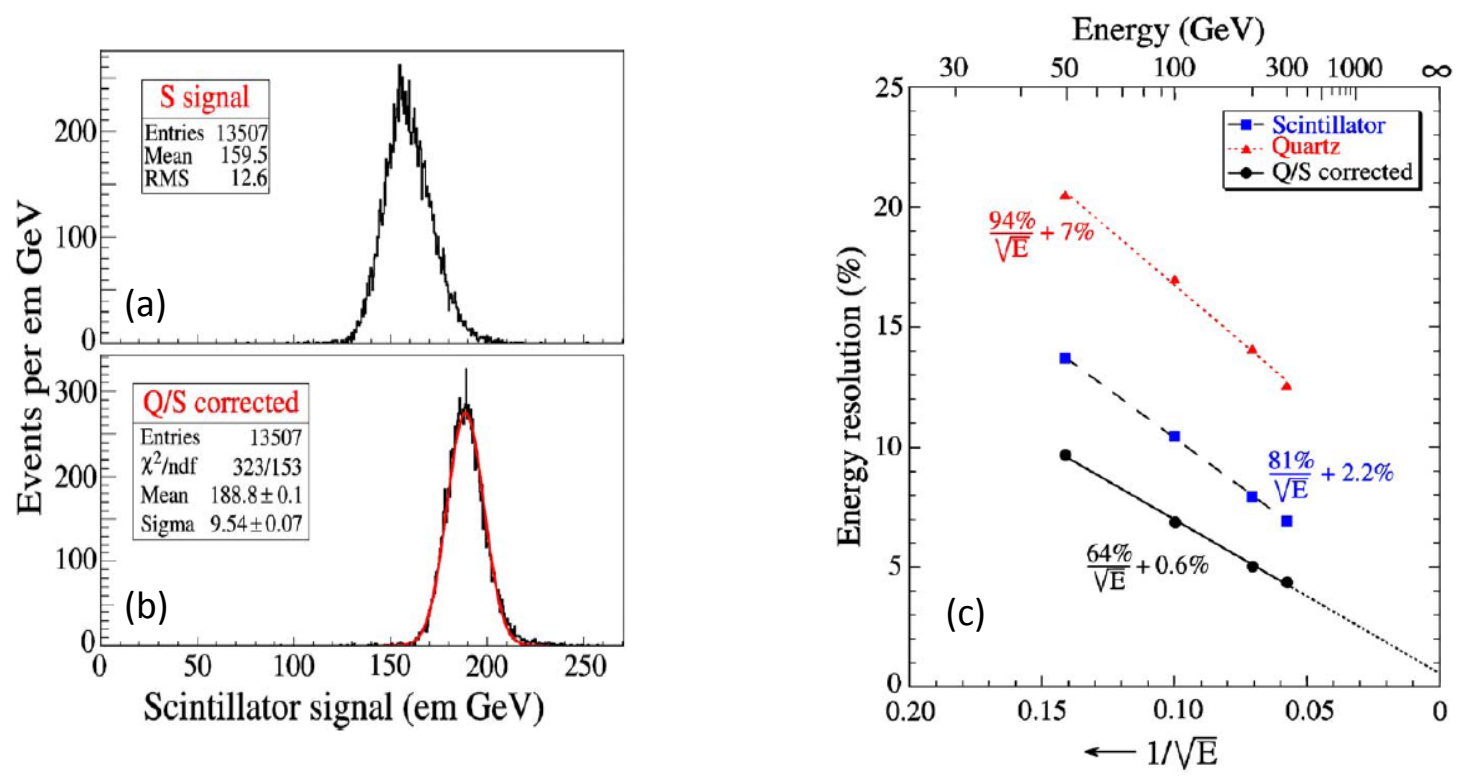

Figure 1: Scintillator signal distribution for $200 \mathrm{GeV}$ "jet" in the DREAM calorimeter before (a) and after (b) the correction based on the measured $C / S$ signal ratio. The "jet" energy resolution as a function of the energy (c), measured with the scintillation fibers and the Cherenkov fibers, and after corrections on the basis of the measured $C / S$ signal ratio. 


\section{The dual readout in crystals.}

In the high $\mathrm{Z}$ scintillating crystals used in homogeneous electromagnetic calorimeters a significant fraction of the light yield comes from Cherenkov radiation ( up to $15 \%$ in PWO). In the last years the DREAM Collaboration has performed extensive studies to extend the dual readout to e.m. calorimeters and many types of crystals (PWO, BGO, BSO, etc.) have been exposed in test beams to evaluate the Cherenkov yields and to optimize the light collection. The peculiar features of the Cherenkov radiation have been exploited to separate the two types of light [2]:

Directionality: the Cherenkov radiation is emitted at an angle $\theta_{C}=\operatorname{arcos}(1 / \beta n)$ with respect to the path of the particle $\left(\theta_{C}=63^{\circ}\right.$ in PWO) then its contribution to the PMT signal strongly depends on the angle of incidence of the particle (fig. 2a). Of course this effect is not observed in the isotropic scintillation light.

Time structure of the signal: the Cherenkov emission is prompt while the scintillation light has a decay constant (fig. 2b).

Spectral distribution: the Cherenkov light has an $1 / \lambda^{2}$ distribution that privilegies wavelengths shorter than in the scintillation. The use of filters can separate the two components (fig.2c).

Polarization: the Cherenkov radiation is polarized then, as pointed out in the test of a BSO crystal [3], a polarizer in front of the photomultiplier can improve the separation from the not polarized scintillation component.

After extensive studies on PWO and BGO crystals, and tests to optimize the crystals with small fractions of dopants (PWO with $0.1 \div 5 \%$ of molybdenum) and different filters [4], a recent analysis has compared a BSO (bismuth silicate) and a BGO crystals of equal dimensions [5]. The scintillation signal is faster in BSO (150 ns decay time) than in BGO (300 ns) and about a factor 4 larger in BGO, but the Cherenkov yield is larger in BSO (a factor 5 with U330 UV filter).
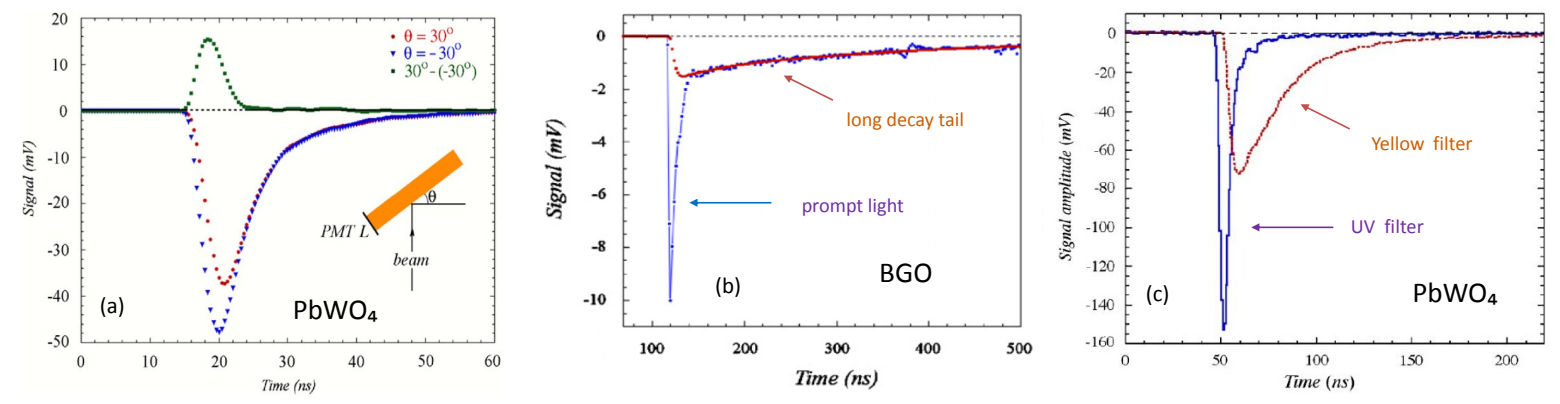

Figure 2: Time structure of the PMT L signals from $50 \mathrm{GeV}$ electrons traversing the PWO crystal at $30^{\circ}$ and $-30^{\circ}$ and their difference (a). Average time structure of the UV signal from $200 \mathrm{GeV} \pi^{+}$with that of the scintillation component contributing to this signal (b). Average time structure of the signals in a PWO crystal (doped with 1\% Mo) with UV and yellow filters (c).

\section{Tests of calorimeter prototypes.}

In particle physics experiments often the e.m. calorimeter realized with crystals is followed by an hadronic calorimeter. The DREAM Collaboration has tested a full size BGO calorimeter 
backed by the original DREAM calorimeter to extend the dual readout to both sections. The e.m. calorimeter is a matrix of $100 \mathrm{BGO}$ crystals, $24 \mathrm{~cm}$ long and tapered $\left(2.4 \times 2.4 \mathrm{~cm}^{2}-3.2 \times 3.2 \mathrm{~cm}^{2}\right)$ from the L3 experiment. A first test was performed in 2009 with a limited readout [6]. In 2010 the matrix was equipped with 16 photomultipliers, the light yield was increased (Scint/Cherenk.: $67 / 8$ ph.e. $/ \mathrm{GeV}$ ), the resolution for $100 \mathrm{GeV}$ electrons was $2.11 \%$ for scintillation and $4.96 \%$ for Cherenkov light. The analysis of the dual readout for the full calorimeter is in progress.

A small calorimeter composed of seven PWO crystals $\left(3 \times 3 \times 20 \mathrm{~cm}^{3}\right.$ doped with $0.3 \%$ Molybdenum (already characterized in [4]) was also exposed on the test beam in 2010. Each crystal was readout at the opposite edges with yellow and UV filters in front of the photomultipliers. The scintillation and Cherenkov components can be separated with an analysis of the signals in wavelength and in time structure. The resolution is $1.3 \%$ and $3 \%$ for scintillation/Cherenkov light.

The possibility to implement the dual readout in a tile sampling calorimeter has also been tested. Tiles of absorber are interleaved with tiles of quartz and scintillator to form two samplings. Each sampling is composed of four times ( $4 \mathrm{~mm}$ lead $+4 \mathrm{~mm}$ quartz $+7 \mathrm{~mm}$ scintillator) square tiles, $9 \times 9 \mathrm{~cm}^{2}$, for a total of 3 radiation lengths. The average muon signals in each sampling is 1.3 photoelectrons for normal impinging beam and 1.6 for $12^{\circ}$ tilted beam. The Cherenkov yield measured for $80 \mathrm{GeV}$ electrons is about 50 ph.e./GeV in both samplings.

\section{The new DREAM calorimeter.}

The Collaboration is now preparing a new fiber calorimeter similar to the DREAM calorimeter but with better performances. Extensive studies have been done to have the largest Cherenkov yield in PMMA clear fibers, the sampling fraction has been increased (5\%, it was $2.6 \%$ in DREAM) and also the quantum efficiency is 50\% larger. The expected Cherenkov yield, about 90 ph.e./GeV in a copper module, is much larger than in the DREAM calorimeter where the dual readout correction was limited by the small Cherenkov yield (8-18 ph.e./GeV). A module of this calorimeter, of dimensions $92 \times 92 \mathrm{~mm}^{2}$ and $2.5 \mathrm{~m}$ in length (about 10 interaction length), is divided in four towers and in each tower there is a separate readout for the Cherenkov and the scintillation signals. In the module there are 46 equal fibers per layer, 46 fiber layers of each type of fiber (scintillating and clear). A first module with lead as absorber was tested in 2010 and a second module was also exposed in the July 2011 test beam. The analysis of the data is in progress.

\section{References}

[1] N.Akchurin, et al.,Nucl. Instr. and Meth. A 537 (2005) 537.

[2] N.Akchurin, et al.,Nucl. Instr. and Meth. A 572 (2007) 215.

[3] N.Akchurin, et al.,Nucl. Instr. and Meth. A 638 (2011) 47.

[4] N.Akchurin, et al.,Nucl. Instr. and Meth. A 621 (2010) 212.

[5] N.Akchurin, et al.,Nucl. Instr. and Meth. A 640 (2011) 91.

[6] N.Akchurin, et al.,Nucl. Instr. and Meth. A 610 (2009) 488. 